\title{
INFORMATIONAL DIVERGENCE BOUNDS FOR AUTHHENTICATION CODES
}

\author{
Andrea Sgarro \\ Department of Mathematics and Computer Science \\ University of Udine \\ I-33100 Udine, Italy \\ Department of Mathematical Sciences \\ University of Trieste \\ I-34100 Trieste, Italy
}

\begin{abstract}
We give an easy derivation of Simmons' lower bound for impersonation games which is based on the non-negativity of the informational divergence. We show that substitution games can be reduced to ancillary impersonation games. We use this fact to extend Simmons' bound to substitution games: the lower bound we obtain performs quite well against those available in the literature.
\end{abstract}

\section{Introduction}

As already observed by the author at Eurocrypt 88 (corridor discussion; of also /1/), a basic lower bound for impersonation games due to Simmons (cf e.g. $/ 2 /$ or $/ 3 /$ ) is a straightforward consequence of the non-negativity of the informational divergence:

$$
D(P, Q)=\sum_{i} p_{i} \log \frac{p_{i}}{q_{i}} \geq 0, D(P, Q)=0 \text { iff } P=Q
$$

$(D(P, Q)$ is called also: discrimination, cross entropy, Kullback-Leibler's number; above $P$ and $Q$ are two probability distributions with the same 
number of components; logs are to a base greater than 1 , e.g. 2 or $e$; for $a>0$ set $\left.0 \log \frac{0}{a}=0 \log \frac{0}{0}=0, a \log \frac{a}{0}=+\infty\right)$. The proof of (1) is itself quite short: one has $\ln x \leq x-1$ with equality iff $x=1$ (ln is strictly concave and lies below its tangent at $x=1$ ); set $x=\frac{q_{i}}{p_{i}}$, multiply by -1 and plug the result into the definition of $D(P, Q)$.

A remarkable divergence is the mutual information $I(Y, Z)$; here $P$ is the joint distribution of the random couple $Y Z$ and $Q$ is the joint distribution obtained by multiplication of the marginal probabilities "as if" $Y$ and $Z$ were independent; (1) implies that the mutual information is non-negative, and is zero iff $Y$ and $Z$ are independent.

Actually, we need conditional divergences: if $Y Z$ and $Y W$ are two random couples over the same product-space the conditional divergence $D(Z, W \mid Y)$ is defined as the weighted average $\sum_{y} \operatorname{Pr}(Y=y) D\left(P_{y}, Q_{y}\right)$, where $P_{y}\left(Q_{y}\right.$, respectively) is the conditional distribution of $Z$ (of $W$, respectively) given $Y=y$. Of course, by (1):

$$
D(Z, W \mid Y) \geq 0, \text { with equality iff } Y Z \simeq Y W
$$

(" $\simeq$ " denotes equality of distributions)

Below we review the easy derivation of Simmons' bound and show that it can be extended to substitution games.

\section{Impersonation games}

Let $Y Z$ be the random couple cryptogram-key. In the impersonation game the opponent chooses a cryptogram $y$ hoping it to be taken as legal. The probability of success is

$$
\operatorname{Pr}\left(Z \in A_{y}\right) \text {, with }
$$




$$
A_{y}=\{z: \operatorname{Pr}(Y=y, Z=z) \neq 0\}
$$

( $A_{y}$ is the set of keys which authenticate $y$ ). The best strategy for the opponent is to choose $y$ as to maximize the probability in (3). Let $P_{I}$ be this maximal probability of success in the impersonation game:

$$
P_{I}=\max _{y} \operatorname{Pr}\left(Z \in A_{y}\right)
$$

Set $\operatorname{Pr}(W=z \mid Y=y)=\operatorname{Pr}\left(Z=z \mid Z \in A_{y}\right)$. Plug this into (2) and recall the definition of the mutual information $I(Y, Z)$ to obtain

$$
E_{Y} \log \operatorname{Pr}\left(Z \in A_{Y}\right) \geq-I(Y, Z)
$$

( $E_{Y}$ denotes expectation.) Noting that $P_{I}$ is the maximal value for the random probability $\operatorname{Pr}\left(Z \in A_{Y}\right)$ one has Simmons' bound (we take binary logs):

$$
P_{I} \geq 2^{-I(Y, Z)}
$$

The conditions for equality are soon found: $Y Z$ and $Y W$ must have the same distribution, and $\operatorname{Pr}\left(Z \in A_{y}\right)$ must be constant in $y$. An authentication system for which (5) holds with equality will be called, in the spirit of Simmons' definition, perfect (actually, perfection as defined by Simmons is more ambitious and covers also the substitution game: ours is perfection with respect to the impersonation game only).

We note that the original derivation of (5) is 'tedious and long', and even the streamlined derivation given in $/ 3 /$ is 'somewhat lengthy' (we are quoting $/ 2 /$ and $/ 3 /$, respectively).

\section{Substitution games}

Assume cryptogram $c$ has been legally sent (and assume that there are at least two cryptograms). The opponent deletes $c$ and sends instead $y \neq c$; 
his probability of success is now:

$$
\operatorname{Pr}\left(Z \in A_{y} \mid Y=c\right)=\operatorname{Pr}\left(Z \in A_{y} \cap A_{c} \mid Y=c\right)
$$

The best strategy is to maximize (6) with respect to $y \neq c$. Let $P_{S}(c)$ be the corresponding maximal probability of success:

$$
P_{S}(c)=\max _{y \neq c} \operatorname{Pr}\left(Z \in A_{y} \cap A_{c} \mid Y=c\right)
$$

Set $a_{c}(z)=\sum_{y \neq c} \operatorname{Pr}(Y=y \mid Z=z) . a_{c}(z)=0$ means that $z$ authenticates only $c$ ( $z$ is a "one-cryptogram key"). Assume for the moment that $a_{c}(z) \neq 0$ for all $z$ in $A_{c}$, and define the random couple $Y_{c} Z_{c}$ as follows:

$$
\begin{gathered}
\operatorname{Pr}\left(Z_{c}=z\right)=\operatorname{Pr}(Z=z \mid Y=c), z \in A_{c} \\
\operatorname{Pr}\left(Y_{c}=y \mid Z_{c}=z\right)=a_{c}(z)^{-1} \operatorname{Pr}(Y=y \mid Z=z), y \neq c
\end{gathered}
$$

Note that in $Y_{c} Z_{c}$ the conditional probabilities of the cryptograms have been "pumped up" so as to sum to 1 ( $c$ is no more there). Let us play the impersonation game for $Y_{c} Z_{c}$ : one has (5) with $Y_{c} Z_{c}$ instead of $Y Z$. If $P_{I}(c)$ denotes the probability of success for the ancillary impersonation game, one has:

$$
\begin{aligned}
& \operatorname{Pr}(c)=\max _{y \neq c} \operatorname{Pr}\left(Z_{c} \in A_{y}^{r}\right)=\max _{y \neq c} \operatorname{Pr}\left(Z \in A_{y}^{c} \mid Y=c\right) \\
& =\max _{y \neq c} \operatorname{Pr}\left(Z \in A_{y}^{c} \cap A_{c} \mid Y=c\right)
\end{aligned}
$$

where $A_{y}^{c}$ is defined as in (4) with $Y_{c} Z_{c}$ instead of $Y Z$. However, as one easily checks, the set of keys which authenticate $y$ in the ancillary impersonation game is the same as the set of keys which authenticate $y$ in the original substitution game:

$$
A_{y}^{c} \cap A_{c}=A_{y} \cap A_{c}
$$


(to see inus, recall definition (4) and observe that $\operatorname{Pr}\left(Z_{c}=z, Y_{c}=y\right) \operatorname{Pr}(Z=$ $z, Y=c) \neq 0$ iff $\operatorname{Pr}(Z=z, Y=y) \operatorname{Pr}(Z=z, Y=c) \neq 0)$. Recalling the definition of $P_{S}(c)$, we obtain the reduction formula we had announced:

$$
P_{I}(c)=P_{S}(c)
$$

Therefore, the lower bound for $P_{I}(c)$ is also a lower bound for $P_{S}(c)$ :

$$
P_{S}(c) \geq 2^{-I\left(Y_{r}, Z_{c}\right)}, Y_{c} Z_{c} \text { defined as in }(7),(8)
$$

By averaging with respect to $\operatorname{Pr}(Y=c)$ one obtains a lower bound for $P_{S}=\sum_{c} \operatorname{Pr}(Y=c) P_{S}(c)$, the overall probability of a successful substitution:

$$
P_{S} \geq \sum_{c} \operatorname{Pr}(Y=c) 2^{-I\left(Y_{c}, Z_{c}\right)}
$$

Equality holds in (10) iff the system $Y_{c} Z_{c}$ is perfect, in (11) iff the systems $Y_{c} Z_{c}$ are perfect for all cryptograms c's.

(10) and (11) have been derived under the assumption $a_{c}(z) \neq 0$. We shall show below (section 4) that this assumption always holds in cases of interest. For reasons of mathematical completeness, however, we shall cover also the case when some of the $a_{c}(z)$ are zero. Then, an extra step is needed. Actually, it is enough to observe that, keys with $a_{c}(z)=0$ being hopeless for the opponent whatever his strategy, he can as well devise his optimal strategy under the assumption that the key $Z$ does not belong to the set $B$ of one-cryptogram keys:

$$
B=\{z: \exists d: \operatorname{Pr}(Y=d \mid Z=Z)=1\}
$$

More formally, choose $y \neq c$ and write the corresponding probability of success as:

$$
\operatorname{Pr}\left(Z \in A_{y} \cap B \mid Y=c\right)+\operatorname{Pr}\left(Z \in A_{y}-B \mid Y=c\right)
$$


The first term in the sum is zero and $\operatorname{Pr}(Z \notin B \mid Y=c)$ does not depend on $y$. Assume the probability just written is not zero (else the whole thing is trivial: substitution attacks on $c$ are unfeasible and so $P_{S}(c)=0$ ); then the purpose of the opponent is to maximize in $y, y \neq c$ :

$$
\operatorname{Pr}\left(z \in A_{y} \mid Y=c, Z \notin B\right)
$$

However, this is the same problem as above ( $\mathrm{cf}(6))$ once the conditioning $Z \notin B$ is imposed. One obtains, in full generality:

$$
P_{S}(c) \geq \operatorname{Pr}(Z \notin B \mid Y=c) 2^{-I\left(Y_{c}, Z_{c}\right)}
$$

Here $Y_{c}$ and $Z_{c}$ are defined as in (7) and (8), only adding the conditioning $Z \notin B$ in the definition of $Z_{c}$. Equality holds iff either the system $Y_{c} Z_{c}$ is perfect, or the lower bound is zero (keys which authenticate $c$ authenticate only $c$; then, to be fastidious, $I\left(Y_{c}, Z_{c}\right)$ is undefined, so that we have to make the convention that zero times an undefined term is zero). By averaging with respect to $\operatorname{Pr}(Y=c)$ one obtains a bound for $P_{S}$, with conditions for equality.

\section{A toy example}

An authentication code is more accurately described as a random triple $X Y Z$ rather than as a random couple $Y Z$ (cf e.g. $/ 2 /$ or $/ 3 /)$ : the term added is the random clearmessage. One assumes $Y$ to be a deterministic function of the couple $X Z$ and $X$ to be a deterministic function of the couple $Y Z$ : these functions correspond to enciphering and (legal) deciphering, respectively; the random key and the random clearmessage are assumed to be independent random variables. By requiring enciphering to be deterministic 
we rule out splitting; formally, our results hold true also in the case with splitting: however, substitution games are meaningful only when substitution of the legal cryptogram implies substitution of the correct clearmessage, that is only when enciphering is deterministic. (Note that often, e.g. in /2/ and $/ 4 /$, the cryptographic-flavoured terms clearmessage, cryptogram and key as used here are replaced by the terms source-state, message (helas!) and encoding rule, respectively; a third alternative, more in keeping with the usage of non-secret coding theory, would be: message, codeword and encoding rule.) A nice way to represent the enciphering and deciphering functions for an authentication code $X Y Z$ is to draw up a matrix whose row-headers and column-headers are the keys and the clearmessages, respectively, and whose entries are the corresponding cryptograms. Of course the $X Y Z$ model is a special case of the $Y Z$ model, and so the lower bounds given above still hold. Actually, since the same cryptogram cannot appear more than once in the same row of the matrix (deciphering is deterministic) one-cryptogram keys are ruled out and only bounds (10) and (11) are needed (we are assuming that the matrix has at least two columns, which is an obvious assumption to make when one plays substitution).

Let us work out a toy example with 2 keys, 2 clearmessages and 3 cryptograns:

$\begin{array}{lll}* & 1 & 2 \\ 1 & 1 & 2 \\ 2 & 3 & 1\end{array}$

We assume equiprobability both for the key and the clearmessage; then $\operatorname{Pr}(Y=1)=\frac{1}{2}, \operatorname{Pr}(Y=2)=\operatorname{Pr}(Y=3)=\frac{1}{4}$. Clearly, the (optimal- 
strategy) probability of successful substitution is $\frac{1}{2}$ if cryptogram 1 is substituted, else is 1 . So the overall probability of a successful substitution is $P_{S}=\frac{3}{4}$. Since this value is returned also by bound (11), as one easily checks, our bound for $P_{S}$ holds here with equality.

In /4/ four lower bounds are given for $P_{S}$ (theorems $2.6,2.9,2.10,2.14$ ); however they return values strictly smaller than $\frac{3}{4}$ for our toy example. A computer search has been performed by $\mathrm{A}$. Mereu, a student at Udine, on a large sample of small-order examples: in no case the bound we have proposed has been strictly beaten.

\section{Final remark}

Formula (9) allows one to reduce substitution games to impersonation games. There is a snag, however. Even if we start by a "complete" authentication code $X Y Z$, the ancillary codes we were able to construct are random couples $Y_{c} Z_{c}$ and not random triples $X_{c} Y_{c} Z_{c}$. We could convert Simmons' bound for impersonation into a bound for substitution only because the former does not involve the random clearmessage in any way.

\section{References}

1/ A. Sgarro, Three-types of perfection in Shannon-theoretic cryptography, Proceedings of a Workshop on Sequences, Positano, June 6-11, 1988

/2/ G.J. Simmons, A survey of information authentication, Proceedings of the IEEE, May 1988, pp. 603-620 
/3/ J.L. Massey, An introduction to contemporary cryptology, Proceedings of the IEEE, May 1988, pp. 533-549

/4/ D.R. Stinson, Some constructions and bounds for authentication codes Journal of Cryptology, 1,1 (1988) pp. 37-52 